\title{
Salidroside and isorhamnetin attenuate urotensin II-induced inflammatory response in vivo and in vitro: Involvement in regulating the RhoA/ROCK II pathway
}

\author{
CHENJING WANG ${ }^{1}$, XIAODONG NAN $^{2}$, SHUYAN PEI $^{1}$, YU ZHAO $^{1}$, \\ XIAOKUN WANG ${ }^{1}$, SHIJIE MA ${ }^{1}$ and GUOYAN MA ${ }^{1}$ \\ ${ }^{1}$ Department of Pharmacology, School of Basic Medical Sciences, Northwest Minzu University Health Science Center, \\ Lanzhou, Gansu 730030; ${ }^{2}$ Intensive Care Unit, Gansu Provincial Corps Hospital of Chinese People's \\ Armed Police Force, Lanzhou, Gansu 730050, P.R. China
}

Received February 16, 2020; Accepted November 11, 2020

DOI: $10.3892 / \mathrm{ol} .2021 .12553$

\begin{abstract}
Urotensin II (UII), a vital vasoconstrictor peptide, causes an inflammatory response in the pathogenesis of atherosclerosis. Previous studies have reported that the Ras homolog gene family, member A (RhoA)/Rho kinases (ROCK) pathway modulates the inflammatory response of the atherosclerotic process. However, to the best of our knowledge, whether the RhoA/ROCK pathway mediates the inflammatory effect of UII has not been previously elucidated. Salidroside and isorhamnetin are two early developed antioxidant Tibetan drugs, both displaying cardioprotective effects against atherosclerosis. Therefore, the aim of the present study was to investigate the protective effects of salidroside, isorhamnetin or combination of these two drugs on the UII-induced inflammatory response in vivo (rats) or in vitro [primary vascular smooth muscle cells (VSMCs)], as well as to examine the role of the RhoA/ROCK pathway in these processes. The levels of inflammatory markers were measured via ELISA. The mRNA and protein expression levels of RhoA and ROCK II were detected using reverse transcription-quantitative PCR assay and western blot analysis. It was demonstrated that salidroside, isorhamnetin and both in combination decreased the levels of the serum pro-inflammatory cytokines TNF- $\alpha$ and IL-1 $\beta$, as well as increased the levels of the anti-inflammatory cytokine IL-10 and macrophage migration inhibitory factor in rats with subacute infusion of UII and in the culture supernatant from primary VSMCs-exposed to UII. Moreover, salidroside,
\end{abstract}

Correspondence to: Dr Chenjing Wang, Department of Pharmacology, School of Basic Medical Sciences, Northwest Minzu University Health Science Center, 1 XibeiXincun, Chengguan, Lanzhou, Gansu 730030, P.R. China

E-mail: wangcj01002@163.com

Key words: salidroside, isorhamnetin, urotensin II, inflammatory response, atherosclerosis, Ras homolog gene family, member A/Rho kinases II pathway isorhamnetin and both in combination attenuated the mRNA and protein expression levels of RhoA and ROCK II in vivo and in vitro, at concentrations corresponding to human therapeutic blood plasma concentrations. Thus, these drugs could inhibit the RhoA/ROCK II pathway under UII conditions. The combination of salidroside and isorhamnetin did not display a stronger inhibitory effect on the inflammatory response and the RhoA/ROCK II pathway compared with salidroside and isorhamnetin in isolation. Collectively, the results indicated that salidroside, isorhamnetin and both in combination inhibited the RhoA/ROCK II pathway, which then attenuated the inflammatory response under UII-induced conditions, resulting in cardioprotection in atherosclerosis.

\section{Introduction}

Atherosclerosis is the leading cause of acute myocardial infarction and stroke, resulting in large global health and economic burdens (1). It has been reported that atherosclerosis is a chronic vascular wall-related inflammatory disease that occurs within the arterial wall (2). Inflammatory factors are mainly divided into three categories: i) Chemokines, including monocyte chemoattractant protein-1 (MCP-1), fractalkine/CX3CR1 and macrophage colony stimulating factor, whose effects are inhibited by macrophage migration inhibition factor (MIF); ii) pro-inflammatory factors, including C-reactive protein, IL-6, IL-1 and TNF- $\alpha$; and iii) anti-inflammatory factors, including IL-10 and TGF- $\beta$ (3). Pharmacological studies have reported that the imbalances between the pro-atherogenic inflammatory response and atheroprotective anti-inflammatory responses serve a key role in the initiation and progression of atherosclerosis (4). Previous in vivo and in vitro experimental data have revealed the key signaling pathways, such as programmed death ligand-1/programmed cell death protein 1 axis, brain-derived neurotrophic factor/tyrosine kinase B signaling pathway (5), Nod-like receptor protein 3 inflammasome/IL-1/IL-18/IL-6 pathway (6) and Toll-like receptor pathways (7), that mediate the inflammatory response, which may be research hotspots and provide potential preventive targets for atherosclerosis. 
Therefore, the treatment of atherosclerosis using anti-inflammatory drugs may be an attractive strategy (8-10).

It has been reported that the Ras homolog gene family, member A (RhoA)/Rho kinases (ROCK) pathway is an important signal transduction system involved in cell proliferation, endothelial dysfunction, oxidative stress, inflammation, vascular remodeling and atherosclerosis (11-13). Previous studies have suggested that various multiple risk factors and pathological mediators of atherosclerosis can activate the RhoA/ROCK pathway to different degrees (14-16), and the inhibition of the RhoA/ROCK pathway could be a therapeutic potential target in the treatment of atherosclerosis (16). Furthermore, the regulatory effect of the RhoA/ROCK pathway on the inflammatory response of the atherosclerotic process has been confirmed by previous finding $(17,18)$. The study from Shimada and Rajagopalan (19) revealed that ROCK mediates lysophosphatidic acid (an inflammatory mediator that is elevated in multiple inflammatory diseases)-induced IL-8 and MCP-1 production in human endothelial cells. The ROCK pathway also contributes to hyperglycemia-activated macrophages, which results in a pro-inflammatory phenotype and eventually contributes to atherosclerosis (17). However, the underlying potential RhoA/ROCK-regulated signaling pathways in inflammatory response under atherosclerosis remain to be elucidated.

Urotensin II (UII), a vasoactive cyclic peptide, and its high-affinity G-protein-coupled receptor UT are both highly expressed in the human cardiovascular system, and UII is involved in the development of cardiovascular homeostasis disease (20,21). Clinical and experimental studies have identified a positive correlation between increased UII levels and the development of atherosclerosis (22-24). UII can enhance the development of aortic atherosclerotic lesions and destabilizes atherosclerotic plaques (25). UII also exerts a pro-inflammatory effect on vascular wall cells in atherosclerosis $(24,26)$. Previous studies have shown that the RhoA/ROCK pathway mediates UII-induced migration of endothelial progenitor cells and the formation of macrophage derived foam cells, suggesting that the RhoA/ROCK pathway may contribute to the UII-induced inflammatory response (27). However, to the best of our knowledge, there are no previous reports on the roles of the RhoA/ROCK pathway in UII-derived inflammatory effects.

Salidroside and isorhamnetin are two early used antioxidant Tibetan drugs, possessing a variety of biological activities such as anti-apoptosis, anti-oxidative stress and anti-inflammation effects $(28,29)$. It has also been reported that salidroside and isorhamnetin exert a cardioprotective effect on the development of atherosclerosis, which is partly dependent on their anti-inflammatory ability $(26,30,31)$. However, the anti-inflammatory or protective properties of salidroside and isorhamnetin against UII-derived inflammatory effects in atherosclerosis, as well as the underlying molecular mechanisms, are yet not fully understood.

Therefore, the present study aimed to investigate the role of the RhoA/ROCK II pathway in the UII-induced inflammatory response, as well as to identify the effects of salidroside and isorhamnetin treatment on the UII-induced inflammatory response and their potential mechanism in vivo and in vitro.

\section{Materials and methods}

Chemicals and reagents. UII (cat. no. U4753), salidroside (cat. no. 05410590) and isorhamnetin (cat. no. 17794) were purchased from Sigma-Aldrich (Merck KGaA). DMEM (cat. no. 11965092) and FBS (cat. no. 16140071) were purchased from Gibco (Thermo Fisher Scientific, Inc.). Anti-RhoA (cat. no. 2117s), anti-ROCK II (cat. no. 9029s) and anti-GAPDH (cat. no. 5174) antibodies were purchased from Cell Signaling Technology, Inc.

Rat modeling and animal treatment. All the experimental procedures were performed in accordance with the National Institutes of Health Guide for the Care and Use of Laboratory Animals (32), and were approved by the Experimental Animal Administration Committee of the School of Basic Medical Sciences, Northwest Minzu University Health Science Center (approval no. XBMZ-YX20130101; March 1, 2013). In total, 120 healthy male Wistar rats (weight, 180-200 g; age, 8 weeks) were provided by Jiangning Qinglongshan Animal Cultivation Farm and were housed under laboratory conditions (temperature $22 \pm 2^{\circ} \mathrm{C}$ with a relative humidity of $40-50 \%$ and natural light-dark cycle time of $12 / 12 \mathrm{~h}$ ) with free access to food and water.

For the preparation of the rat model, rats were anaesthetized with pentobarbital sodium [60 $\mathrm{mg} / \mathrm{kg}$; intraperitoneal (i.p.)] and osmotic mini-pumps (Alzet Model 2006D; Durect Corporation) were loaded with either UII or saline alone (vehicle). The rats were randomly divided into eight groups: i) Normal control (saline alone, equal amount as the UII group, $n=15)$; ii) UII group [rats were subcutaneously injected with UII (10 $\mathrm{ng} / \mathrm{kg} / \mathrm{min})$ for 7 consecutive days, $\mathrm{n}=15$ ]; ii) Salidroside $(12 \mathrm{mg} / \mathrm{kg})+$ UII group, $(\mathrm{n}=15)$; iv) Salidroside $(24 \mathrm{mg} / \mathrm{kg})+$ UII group, $(\mathrm{n}=15)$; v) Isorhamnetin $(12 \mathrm{mg} / \mathrm{kg})+$ UII group, $(\mathrm{n}=15)$; vi) Isorhamnetin $(24 \mathrm{mg} / \mathrm{kg})+$ UII group, $(\mathrm{n}=15)$; vii) Salidroside + isorhamnetin (Both, $12 \mathrm{mg} / \mathrm{kg})+$ UII group, $(\mathrm{n}=15)$; and viii) Salidroside + isorhamnetin (Both, $24 \mathrm{mg} / \mathrm{kg})+$ UII group, $(\mathrm{n}=15)$. The doses of salidroside and isorhamnetin in vivo and in vitro were chosen in accordance with the previous literatures (33-36).

Isolation and identification of primary vascular smooth muscle cells (VSMCs). A total of 8 adult male Wistar rats (age, 10 weeks; weight, 250-350 g) housed under the same aforementioned laboratory conditions were heparinized (4 IU/g; i.p.) and then euthanatized via pentobarbital sodium (100 mg/kg; i.p.) administration. The procedure for VSMCs isolation was performed in accordance with a previously described protocol (31). The aorta was immediately collected, placed into $75 \%$ (v/v) alcohol, dissected into sections (length, $3 \mathrm{~cm}$ ) and subsequently placed in PBS. After removing fibroblasts that were present in the tunica externa (the external third of the vessel wall thickness) using forceps, the residual vessels were longitudinally cut and the tunica interna was scraped off, leaving the tunica media. Then, the tunica media was washed with DMEM, cut into sections $\left(1 \mathrm{~mm}^{3}\right)$ and maintained in DMEM containing $10 \%(\mathrm{v} / \mathrm{v}) \mathrm{FBS}$ at $37^{\circ} \mathrm{C}$ in a Heraeus $5 \% \mathrm{CO}_{2}$ incubator (Thermo Fisher Scientific, Inc.). The morphology of cultured VSMCs was observed by phase contrast microscopy at passages 3-8 at room temperature. The identification of VSMCs 
was performed using a Histostain-streptavidin-peroxidase kit (rabbit; cat. no. SP-0023; Beijing Biosynthesis Biotechnology Co., Ltd.) according to the manufacturer's protocol. Briefly, the third generation of VSMCs was inoculated into a $100-\mathrm{mm}$ culture. When the cells grew to near fusion state, the cover glass was removed and the cells were fixed with $4 \%$ freshly prepared cooled neutral paraformaldehyde buffer at room temperature for 15 min and washed with PBS. Subsequently, after blocking non-specific binding sites with 5\% BSA Blocking Reagent included in the aforementioned immunohistochemical kit at $37^{\circ} \mathrm{C}$ for $30 \mathrm{~min}$, rabbit anti-a smooth muscle actin polyclonal antibodies (cat. no. bs-0189R; Beijing Biosynthesis Biotechnology Co., Ltd.) were incubated with the cells overnight at $4^{\circ} \mathrm{C}$. Next, the slides were incubated with secondary antibodies for $1 \mathrm{~h}$ at room temperature and subsequently stained with a-smooth muscle actin antibody included in the immunohistochemical kit for $10 \mathrm{~min}$ at room temperature. The cells were visualized using a laser confocal microscope (Leica Microsystems GmbH; magnification, x400).

Cell culture and treatment. VSMCs were cultured in DMEM supplemented with $10 \%$ FBS in a humidified atmosphere with $5 \% \mathrm{CO}_{2}$ at $37^{\circ} \mathrm{C}$. To investigate the effects of UII on VSMCs, cells were treated with different concentration of UII $\left(10^{-9}\right.$, $10^{-8}, 10^{-7}$ and $10^{-6} \mathrm{~mol} / \mathrm{l}$ ) for 24 hat $37^{\circ} \mathrm{C}$. To demonstrate the impacts of salidroside and isorhamnetin on VSMCs exposed to UII, cells were pretreated with salidroside $(1,3$ or $10 \mu \mathrm{M})$, isorhamnetin $(1,3$ or $10 \mu \mathrm{M})$ or both salidroside (3 or $10 \mu \mathrm{M})$ and isorhamnetin $(3$ or $10 \mu \mathrm{M})$ for $1 \mathrm{~h}$, followed by treatment with UII $\left(10^{-6} \mathrm{~mol} / \mathrm{l}\right)$ for 24 hat $37^{\circ} \mathrm{C}$.

Measurement of inflammatory markers in the culture supernatant using ELISA. VSMCs were seeded into a 6-well plate at a density of $1 \times 10^{6}$ cells $/ \mathrm{ml}$. After incubation for $24 \mathrm{~h}$ as aforementioned, the culture supernatants were collected, centrifuged at 1,000 $\mathrm{x} \mathrm{g}$ for $5 \mathrm{~min}$ at room temperature, and used to assess the levels of TNF- $\alpha$ (Nanjing Jiancheng Bioengineering Research Institute; cat. no. H052), IL-1 $\beta$ (Nanjing Jiancheng Bioengineering Research Institute; cat. no. H002), IL-10 (Nanjing Jiancheng Bioengineering Research Institute; cat. no. H009) and MIF (BioLegend ${ }^{\circledR}$ Legend Max TM Human Active MIF; cat. no. 438408; BioLegend, Inc.) with ELISA kits, according to manufacturer's instructions. The supernatants $(500 \mu \mathrm{M})$ were seeded into enzyme labelling 96-well plate and anti-TNF- $\alpha$, IL-1 $\beta$, IL-10 and MIF antibodies $(200 \mu \mathrm{M})$ included in the kits were added, respectively. Following incubation for $2 \mathrm{~h}$ at room temperature, horseradish peroxidase (HPR)-labelled secondary antibody included in the kit $(200 \mu \mathrm{M})$ was added to for $1 \mathrm{~h}$ at room temperature. The absorbance value at $450 \mathrm{~nm}$ was determined using a Multiskan Microplate reader (Thermo Fisher Scientific, Inc.).

Reverse transcription-quantitative $P C R(R T-q P C R)$ assay. The total RNA in each group was extracted with TRIzol ${ }^{\circledR}$ reagent (Invitrogen; Thermo Fisher Scientific, Inc.; cat. no. 15596018), and reverse-transcribed into cDNA using a PrimeScript RT Reagent kit (Takara Bio, Inc.; cat. no. RR037B). The RT conditions were $10 \mathrm{~min}$ at $25^{\circ} \mathrm{C}, 45 \mathrm{~min}$ at $48^{\circ} \mathrm{C}$ and a final step for $6 \mathrm{~min}$ at $95^{\circ} \mathrm{C}$. RT-qPCR was performed using Platinum SYBR Green qPCR SuperMix (Invitrogen; Thermo Fisher
Scientific, Inc.; cat. no. 11744100) on an ABI Prism 7500 system (MP Biomedicals) according to the manufacturer's instructions. The amplification conditions were as follows: Initial denaturation at $95^{\circ} \mathrm{C}$ for $1 \mathrm{~min}$, followed by 40 cycles of $95^{\circ} \mathrm{C}$ for $5 \mathrm{sec}, 60^{\circ} \mathrm{C}$ for $15 \mathrm{sec}$ and $72^{\circ} \mathrm{C}$ for $30 \mathrm{sec}$. GAPDH was used as the endogenous control. The mRNA expression levels of RhoA and ROCK II were normalized to GAPDH using the $2^{-\Delta \Delta \mathrm{Cq}}$ equation (37). The primer sequences used were as follows: RhoA forward, 5'-TCGGAATGATGAGCA CACAA-3' and reverse, 5'-GCTTCACAAGATGAGGCAC-3'; ROCK II forward, 5'-CAGCAACTTTGACGACATTGAG-3' and reverse, 5'-AGATTTGCACTTCTGTTCCAGC-3'; and GAPDH forward, 5'-ACGGCAAGTTCAACGGCACAG-3' and reverse, 5'-GACGCCAGTAGACTCCACGACA-3'.

Western blot analysis. After the indicated treatments, VSMCs were harvested, lysed in RIPA buffer (Beyotime Institute of Biotechnology; cat. no. P0013K) containing 1\% (V/V) PMSF (Beyotime Institute of Biotechnology; cat. no. ST506) on ice for $30 \mathrm{~min}$, and then centrifuged at $13,000 \mathrm{x} \mathrm{g}$ for $10 \mathrm{~min}$ at $4^{\circ} \mathrm{C}$. The protein concentration was confirmed using a BCA Protein Assay kit (Beyotime Institute of Biotechnology; cat. no. P0012). Equal amounts of protein (30 $\mu \mathrm{g} /$ lane) were separated on $12 \%$ SDS-PAGE, transferred onto PVDF membranes (EMD Millipore; cat. no. IPVH00010) and blocked with blocking buffer [0.1\% Tween-20 in TBS (TBS-T) supplemented with $5 \%$ fat-free milk] for $2 \mathrm{~h}$ at room temperature. After washing with TBS-T buffer, the membranes were incubated with anti-RhoA, anti-ROCK II and anti-GAPDH antibodies $(1: 2,000)$ overnight at $4^{\circ} \mathrm{C}$. GAPDH was used as a loading control. Then, the membranes were incubated with HRP-conjugated secondary antibody (1:5,000; cat. no. 7077; Cell Signaling Technology, Inc.) for $2 \mathrm{~h}$ at room temperature. The immunoreactive bands were visualized using a chemiluminescence imaging analysis system (cat. no. 32106; Pierce; Thermo Fisher Scientific, Inc.). The densities of protein expression were semi-quantified using Bio-Rad ChemiDoc XRS (version 4.3.0;Bio-Rad Laboratories, Inc.).

Statistical analysis. Data are presented as the mean \pm SD from $\geq 3$ different experiments. Data were analyzed using SPSS.18 software (SPSS, Inc.). Comparison among multiple relevant groups was performed using a one-way ANOVA followed by Bonferroni's multiple comparison test. $\mathrm{P}<0.05$ was considered to indicate a statistically significant difference.

\section{Results}

Salidroside, isorhamnetin and both in combination decrease the levels of serum inflammatory cytokines after subacute infusion of UII in rats. The results of ELISA suggested that compared with the control group, after subacute infusion of UII (10 ng/kg/min) for 7 days, the levels of pro-inflammatory cytokines TNF- $\alpha$ (Fig. 1A) and IL-1 $\beta$ (Fig. 1B) were significantly upregulated, while the levels of anti-inflammatory cytokine IL-10 (Fig. 1C) and MIF (Fig. 1D) were significantly downregulated in rat serum. However, compared with UII group, salidroside (12 and $24 \mathrm{mg} / \mathrm{kg}$ ), isorhamnetin $(24 \mathrm{mg} / \mathrm{kg}$ ) and the combination of salidroside (12 or $24 \mathrm{mg} / \mathrm{kg}$ ) and isorhamnetin (12 or $24 \mathrm{mg} / \mathrm{kg}$ ) significantly attenuated the 

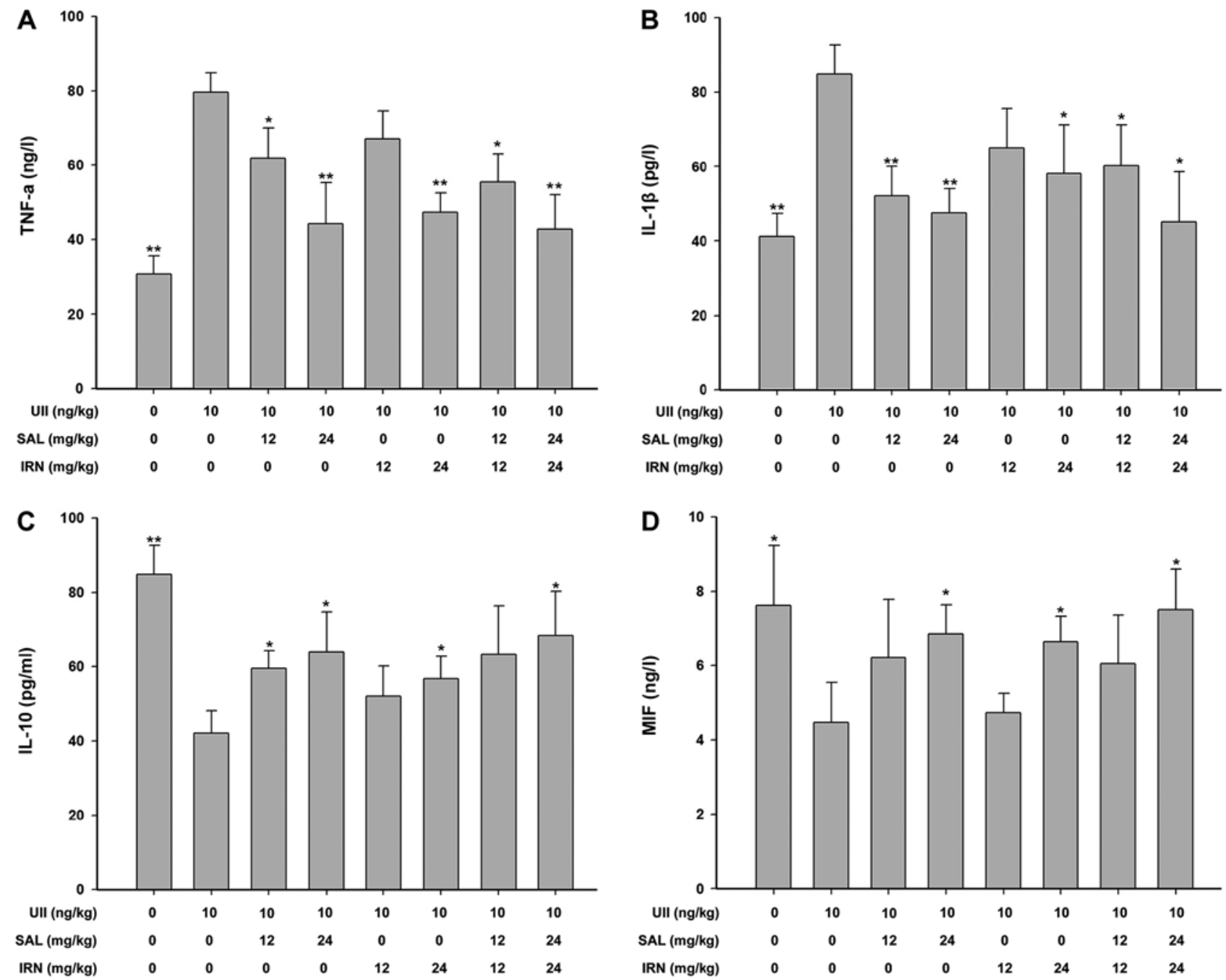

Figure 1. Effects of SAL and IRN on inflammatory cytokines in the serum of rats following UII treatment. Levels of (A) TNF- $\alpha$, (B) IL-1 $\beta$, (C) IL-10 and (D) MIF in the serum of rats were analyzed via ELISA. Data are from $\geq 3$ independent experiments and presented as the mean $\pm \mathrm{SD}$. ${ }^{*} \mathrm{P}<0.05$, ${ }^{* *} \mathrm{P}<0.01$ vs. UII group. MIF, macrophage migration inhibitory factor; UII, Urotensin II; SAL, salidroside; IRN, isorhamnetin.

levels of TNF- $\alpha$ (Fig. 1A) and IL-1 $\beta$ (Fig. 1B). Moreover, salidroside (12 and $24 \mathrm{mg} / \mathrm{kg})$, isorhamnetin $(24 \mathrm{mg} / \mathrm{kg})$ and the combination of salidroside $(24 \mathrm{mg} / \mathrm{kg})$ and isorhamnetin $(24 \mathrm{mg} / \mathrm{kg})$ significantly increased the level of IL-10 compared with UII group (Fig. 1C). It was also identified that salidroside $(24 \mathrm{mg} / \mathrm{kg})$, isorhamnetin $(24 \mathrm{mg} / \mathrm{kg})$ and the combination of salidroside $(24 \mathrm{mg} / \mathrm{kg})$ and isorhamnetin $(24 \mathrm{mg} / \mathrm{kg})$ significantly promoted the level of MIF compared with UII group (Fig. 1D) in the serum of rats. The combination of salidroside $(24 \mathrm{mg} / \mathrm{kg})$ and isorhamnetin $(24 \mathrm{mg} / \mathrm{kg})$ did not exert a higher effect compared with salidroside or isorhamnetin alone (one-way ANOVA followed by Bonferroni's multiple comparison test). These results indicated that salidroside, isorhamnetin and both in combination protected rats against the UII-induced inflammatory response.

Salidroside, isorhamnetin and both in combination inhibit the RhoA/ROCK II pathway in the thoracic aorta of rats following subacute infusion of UII. Previous studies have reported that the RhoA/ROCK pathway can be activated by various atherosclerosis-related risk factors to different degrees, and may participate in the pathogenesis of atherosclerosis $(11,38)$. Thus, the present study investigated the effects of salidroside and isorhamnetin on the RhoA/ROCK II pathway in UII-treated rats. The results demonstrated that subacute infusion of UII significantly increased the mRNA expression levels of RhoA (Fig. 2A) and ROCK II (Fig. 2B) in thoracic aorta compared with control group. Furthermore, administration of salidroside (12 and $24 \mathrm{mg} / \mathrm{kg}$ ), isorhamnetin $(24 \mathrm{mg} / \mathrm{kg}$ ) and the combination of salidroside (12 or $24 \mathrm{mg} / \mathrm{kg}$ ) and isorhamnetin (12 or $24 \mathrm{mg} / \mathrm{kg}$ ) significantly reduced the mRNA expression level of RhoA (Fig. 2A), while administration of salidroside $(24 \mathrm{mg} / \mathrm{kg})$, isorhamnetin $(24 \mathrm{mg} / \mathrm{kg})$ and the combination of salidroside (12 or $24 \mathrm{mg} / \mathrm{kg}$ ) and isorhamnetin (12 or $24 \mathrm{mg} / \mathrm{kg}$ ) significantly decreased the mRNA expression level of ROCK II (Fig. 2B), compared with the UII group.

Western blot analysis results further indicated that UII significantly increased the protein expression levels of RhoA (Fig. 2C) and ROCK II (Fig. 2D) in thoracic aorta of rats, compared with the control group. However, these impacts were mitigated by salidroside ( 12 or $24 \mathrm{mg} / \mathrm{kg}$ ), isorhamnetin $(24 \mathrm{mg} / \mathrm{kg})$, both salidroside $(12 \mathrm{mg} / \mathrm{kg})$ and isorhamnetin $(12 \mathrm{mg} / \mathrm{kg})$, and both salidroside $(24 \mathrm{mg} / \mathrm{kg})$ and isorhamnetin $(24 \mathrm{mg} / \mathrm{kg}$ ) in combination. When the drug concentration 
A

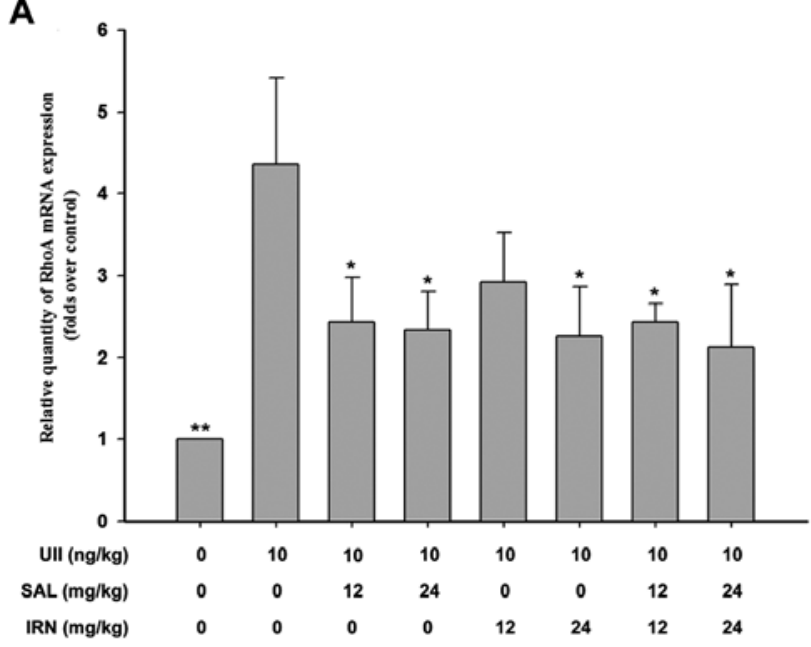

C
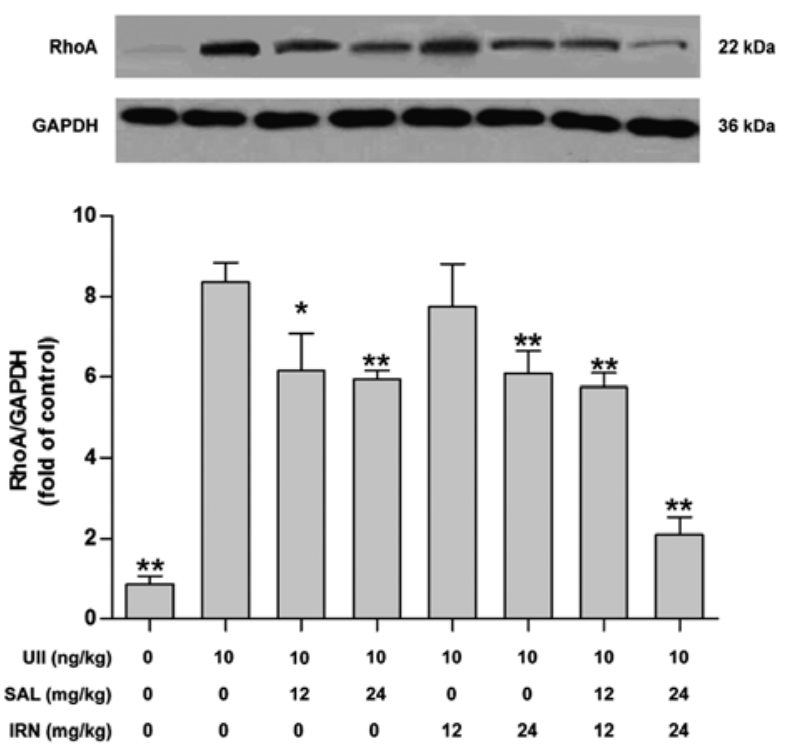

B

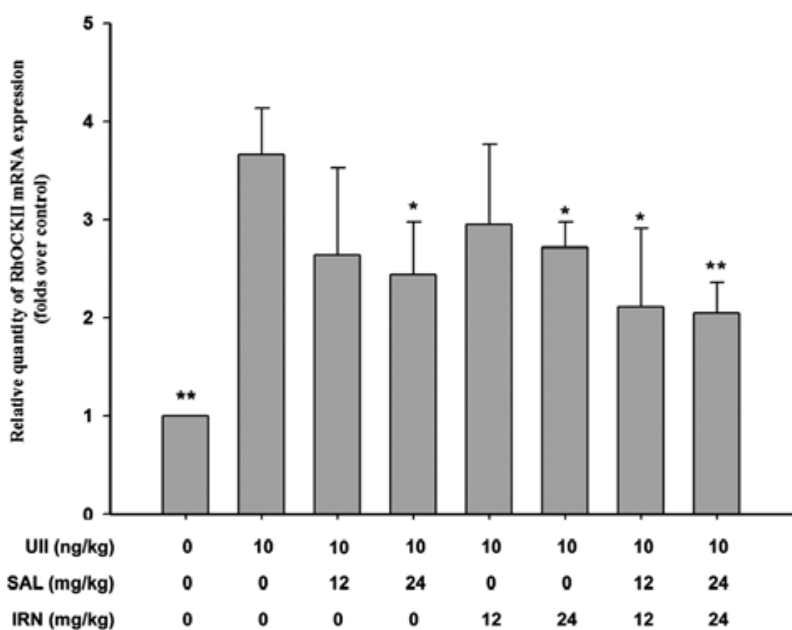

D
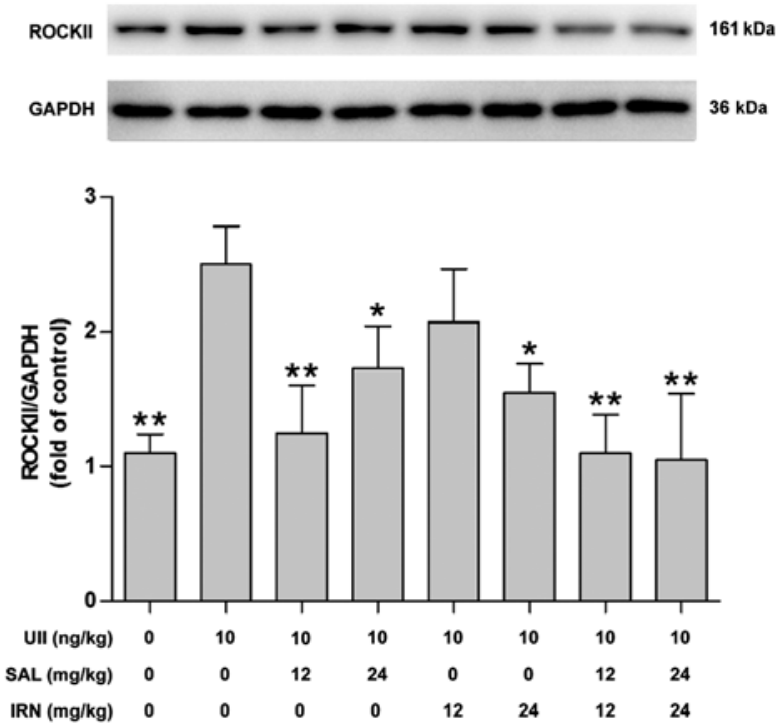

Figure 2. Effects of SAL and IRN on the RhoA/ROCK II pathway in the thoracic aorta of rats following subacute infusion of UII. mRNA expression levels of (A) RhoA and (B) ROCK II were measured via reverse transcription-quantitative PCR. Protein expression levels of (C) RhoA and (D) ROCK II were detected using western blot analysis. Data are from $\geq 3$ independent experiments and presented as the mean $\pm \mathrm{SD}$. ${ }^{*} \mathrm{P}<0.05,{ }^{* *} \mathrm{P}<0.01$ vs. UII group. RhoA, Ras homolog gene family, member A; ROCK, Rho kinases; UII, Urotensin II; SAL, salidroside; IRN, isorhamnetin.

was low, the combination of the two drugs could inhibit the RhoA/ROCK II pathway under UII-induced conditions. Moreover, the inhibitory effects of the combination of salidroside and isorhamnetin on the RhoA/ROCK II pathway were not more effective compared with salidroside or isorhamnetin alone (one-way ANOVA followed by Bonferroni's multiple comparison test). These results suggested that salidroside, isorhamnetin and both in combination inhibited the RhoA/ROCK II pathway, which may lead to the inhibition of UII-induced inflammation.

UII promotes the inflammatory response and the RhoA/ROCK II pathway in primary VSMCs. VSMCs were isolated from male Wistar rats. Under a phase contrast microscope, a small number of cells emerged from the surrounding of the tissue block (rat thoracic aorta) after 4-5 days of culture (Fig. 3A-1). After 10-12 days of culture, the cells in the local bundles were arranged in parallel, and some cells overlapped in multiple layers, demonstrating typical ups and downs of 'peaks' and 'valleys' (Fig. 3A-2). In the third generation of VSMCs, actin immunocytochemical staining identified that $>99 \%$ of the cells were positive; the cytoplasm was brownish yellow and the nucleus was not stained (Fig. 3A-3). These results indicated that VSMCs were successfully extracted.

Subsequently, the effects of UII on the inflammatory response in VSMCs were detected via ELISA. With the increased concentration of UII $\left(10^{-9}, 10^{-8}, 10^{-7}\right.$ and $\left.10^{-6} \mathrm{~mol} / \mathrm{l}\right)$ stimulation for $24 \mathrm{~h}$, the levels of the anti-inflammatory cytokines IL-10 (Fig. 3B) and MIF (Fig. 3C) were significantly decreased. In addition, RT-qPCR results demonstrated that UII $\left(10^{-8}, 10^{-7}\right.$ and $\left.10^{-6} \mathrm{~mol} / \mathrm{l}\right)$ treatment for $24 \mathrm{~h}$ induced significant increases in the mRNA expression levels of RhoA (Fig. 3D) and ROCK II (Fig. 3E) in VSMCs. Western blot analysis (Fig. 3F) results identified that UII $\left(10^{-8}, 10^{-7}\right.$ and $\left.10^{-6} \mathrm{~mol} / \mathrm{l}\right)$ stimulation for $24 \mathrm{~h}$ significantly increased the expression of RhoA (Fig. 3G), and UII $\left(10^{-7}\right.$ and $\left.10^{-6} \mathrm{~mol} / \mathrm{l}\right)$ significantly increased the expression of ROCK II (Fig. 3H) in VSMCs. As UII $\left(10^{-6} \mathrm{~mol} / \mathrm{l}\right)$ treatment for $24 \mathrm{~h}$ significantly upregulated the 
A

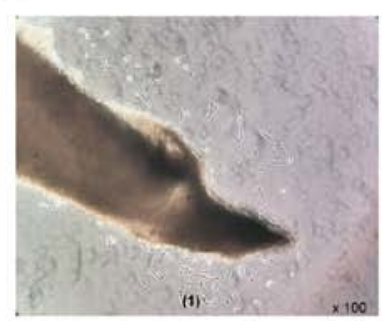

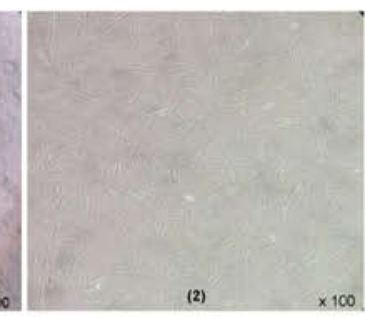
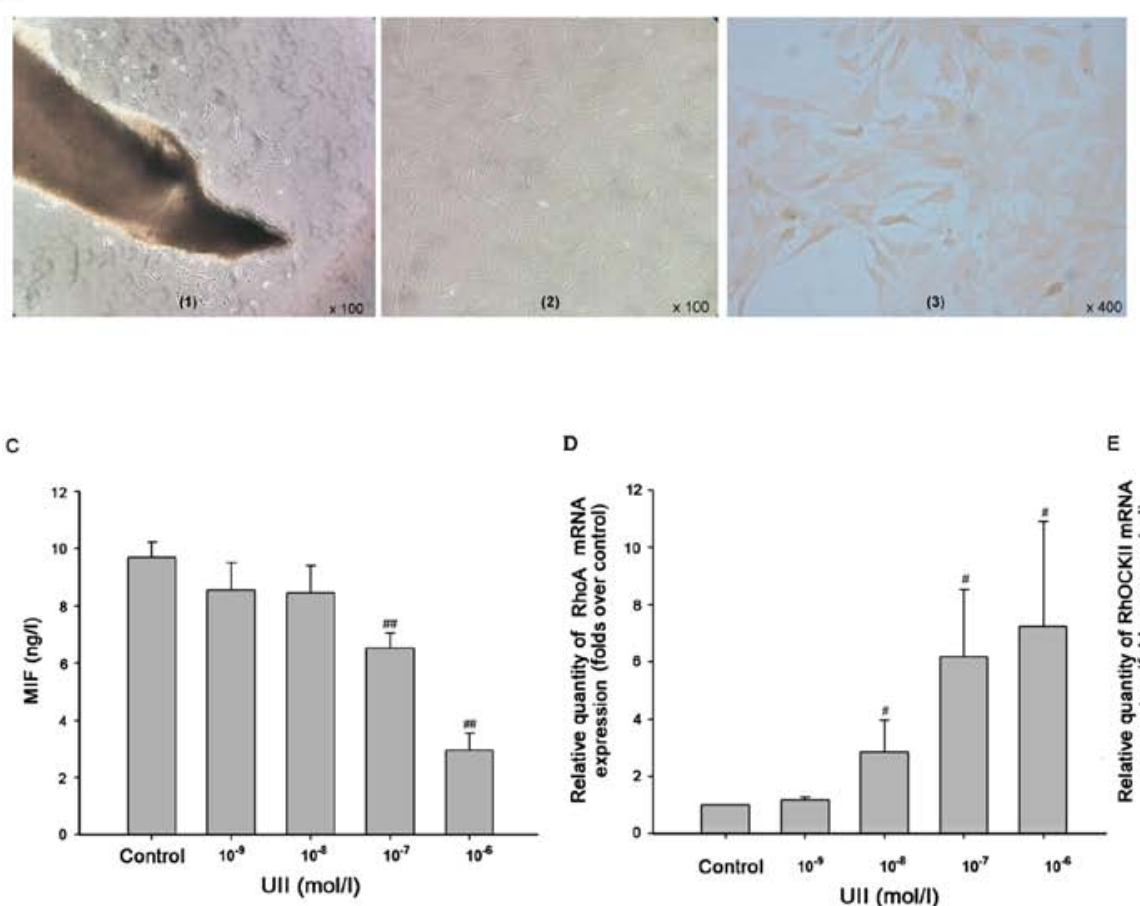

$\mathrm{F}$

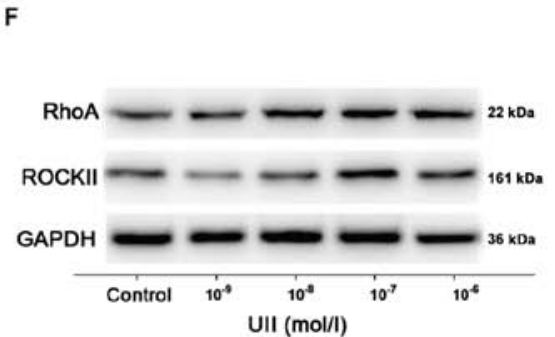

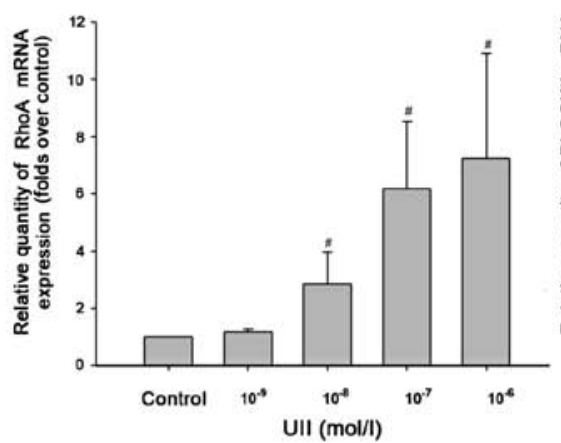

$E$

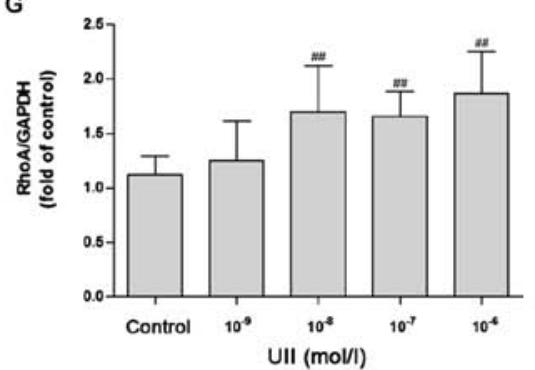

B

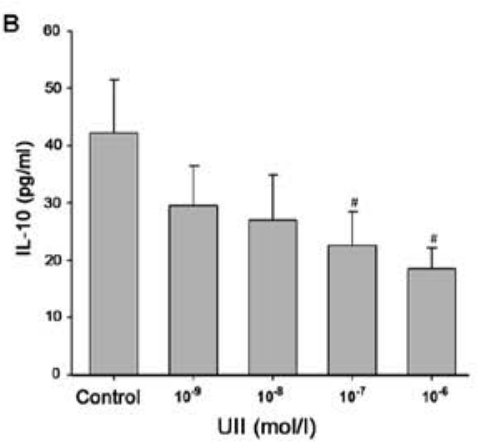

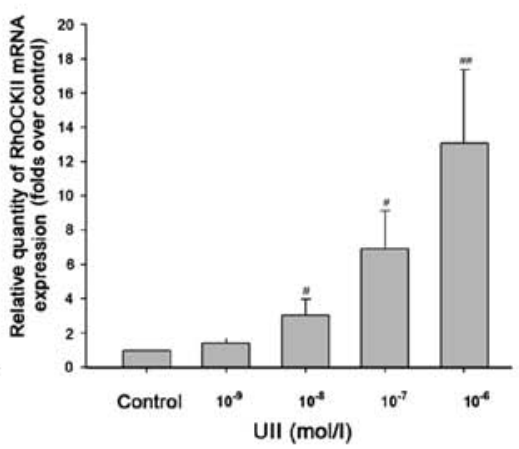

H

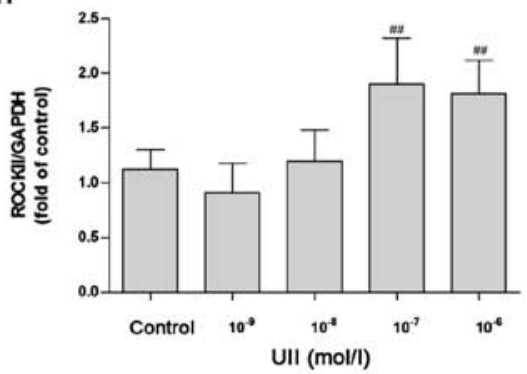

Figure 3. Effects of UII on the inflammatory response and the RhoA/ROCK II pathway in primary vascular smooth muscle cells. (A) Observation of cell morphology. (A-1) After 4-5 days of culture, cell morphology was observed under the phase contrast microscope (magnification, x100). (A-2) After 10-12 days of culture, cell morphology was observed under an invert microscope (magnification, x100). (A-3) $\alpha$-actin immunocytochemical staining (magnification, x400). Inflammatory markers (B) IL-10 and (C) MIF in the culture supernatant were measured via ELISA. mRNA expression levels of (D) RhoA and (E) ROCK II were detected using reverse transcription-quantitative PCR. Protein expression levels of (G) RhoA and (H) ROCK II were detected using (F) western blot analysis. Data are from $\geq 3$ independent experiments and presented as the mean $\pm \mathrm{SD} .{ }^{\#} \mathrm{P}<0.05,{ }^{\# \#} \mathrm{P}<0.01$ vs. control. RhoA, Ras homolog gene family, member $\mathrm{A}$; ROCK, Rho kinases; UII, Urotensin II; MIF, macrophage migration inhibitory factor; SAL, salidroside; IRN, isorhamnetin.

protein expression levels of RhoA and ROCK II in VSMCs, this treatment regimen was selected for subsequent experiments. Taken together, it was suggested that UII stimulation promoted the inflammatory response by enhancing the RhoA/ROCK II pathway in primary VSMCs.

Salidroside, isorhamnetin and both in combination attenuate the UII-induced inflammatory response and inhibit the RhoA/ROCK II pathway in VSMCs. Next, in order to demonstrate the inhibitory effects of salidroside and isorhamnetin on inflammatory responses in VSMCs stimulated with UII, VSMCs were pretreated with salidroside, isorhamnetin or both in combination for $1 \mathrm{~h}$ followed by treatment with UII $\left(10^{-6} \mathrm{~mol} / \mathrm{l}\right)$ for $24 \mathrm{~h}$. Compared with the control group. UII treatment significantly increased the TNF- $\alpha$ (Fig. 4A) and IL-1 $\beta$ (Fig. 4B) levels, and decreased the IL-10 level (Fig. 4C). Moreover, compared with the UII treatment group, pretreatment with salidroside (3 and $10 \mu \mathrm{M})$, isorhamnetin
(3 and $10 \mu \mathrm{M}$ ) and both in combination reduced TNF- $\alpha$ level (Fig. 4A), while pretreatment with salidroside $(10 \mu \mathrm{M})$, isorhamnetin $(10 \mu \mathrm{M})$ and both in combination reduced IL-1 $\beta$ level (Fig. 4B) in UII-treated VSMCs. It was also found that pretreatment with salidroside $(3$ and $10 \mu \mathrm{M})$, isorhamnetin $(1$, 3 and $10 \mu \mathrm{M}$ ) and both in combination increased the IL-10 level (Fig. 4C) in UII-treated VSMCs. The low concentration of salidroside $(1 \mu \mathrm{M})$ had no effect on the level of inflammatory-related factors in UII-treated VSMCs.

The results suggested that pretreatment with salidroside (3 and $10 \mu \mathrm{M})$, isorhamnetin $(10 \mu \mathrm{M})$ and a combination of salidroside and isorhamnetin attenuated the UII-induced increases in the mRNA expression level of RhoA (Fig. 4D). In addition, pretreatment with salidroside ( 3 and $10 \mu \mathrm{M}$ ), isorhamnetin ( 3 and $10 \mu \mathrm{M})$ and both in combination attenuated the UII-induced the increases in the mRNA expression levels of ROCK I (Fig. 4E) and ROCK II (Fig. 4F). Western blotting results (Fig. 4G) also identified that salidroside $(10 \mu \mathrm{M})$, isorhamnetin $(10 \mu \mathrm{M})$ and 

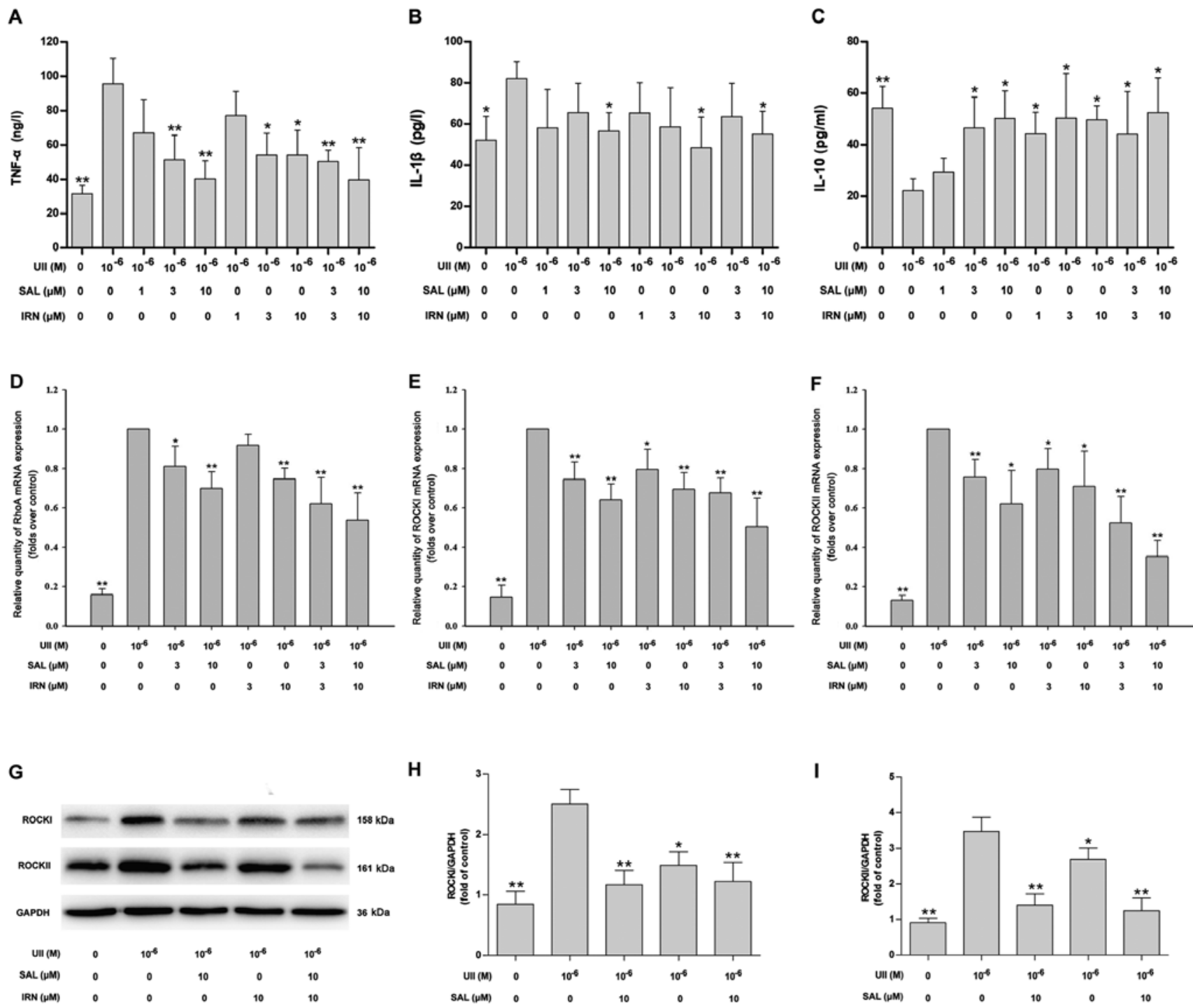

$\mathbf{H}$

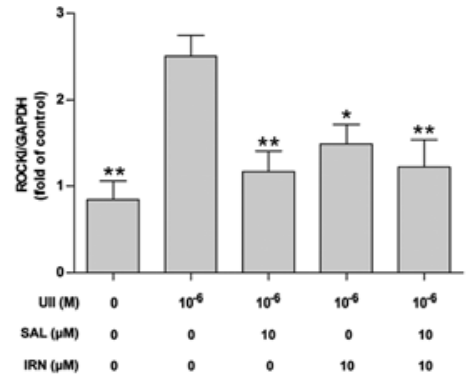

I

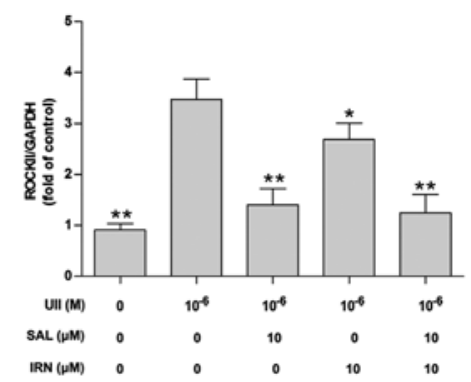

Figure 4. Effects of SAL and IRN on inflammatory response and the RhoA/ROCKII pathway in VSMCs stimulated with UII. (A) VSMCs were pretreated with SAL or IRN $(1,3$ and $10 \mu \mathrm{M})$ alone or SAL and IRN $(3$ and $10 \mu \mathrm{M})$ in combination for $1 \mathrm{~h}$ followed by treatment with UII $\left(10^{-6} \mathrm{~mol} / 1\right)$ for $24 \mathrm{~h}$. Quantitative analysis of (A) TNF- $\alpha$, (B) IL-1 $\beta$ and (C) IL-10 levels via ELISA. mRNA expression levels of (D) RhoA, (E) ROCK I and (F) ROCK II were detected via reverse transcription-quantitative PCR. (G) Western blot analysis of the protein expression levels of (H) ROCK I and (I) ROCK II were measured using. Data are from $\geq 3$ independent experiments and presented as the mean $\pm \mathrm{SD}$. ${ }^{*} \mathrm{P}<0.05,{ }^{* *} \mathrm{P}<0.01$ vs. UII group. MIF, macrophage migration inhibitory factor; UII, Urotensin II; RhoA, Ras homolog gene family, member A; ROCK, Rho kinases; SAL, salidroside; IRN, isorhamnetin; VSMCs, vascular smooth muscle cells.

both in combination reversed UII-induced upregulation of ROCK I (Fig. 4H) and ROCK II (Fig. 4I) protein expression levels in VSMCs. There were no statistically significant differences between the combination of drugs and with salidroside or isorhamnetin alone (one-way ANOVA followed by Bonferroni's multiple comparison test). Collectively, it was indicated that salidroside, isorhamnetin and both in combination mitigated the UII-induced inflammatory response in VSMCs partly by inhibiting the RhoA/ROCK pathway.

\section{Discussion}

The present findings indicated that UII stimulation resulted in an inflammatory response along with an increase in the RhoA/ROCK pathway activation in vivo and in vitro. It was also demonstrated that salidroside, isorhamnetin and both in combination could elicit inhibitory effects on the UII-induced inflammatory response, at least partially by attenuating the RhoA/ROCK pathway.

Previous studies have reported that UII exerts vasculopathic and vasculoprotective effects, and contributes to the pathogenesis of atherosclerosis $(26,39,40)$. UII promotes VSMCs proliferation by activating reactive oxygen species (ROS) and MAPK signaling pathways, which results in vascular remodeling (41). UII also participates in macrophage activation via UII receptor/ROS/Akt pathways in RAW264.7 macrophages (26). Furthermore, UII can promote NAD $(\mathrm{P}) \mathrm{H}$ oxidase-induced reactive oxygen production from a variety of inflammatory cells, resulting in activated NF- $\mathrm{NB}(42,43)$, which is the most important downstream event involved in the signal transduction pathway of various inflammatory factors in the process of vascular injury and atherosclerosis (44-46). These results provide evidence for the inflammatory effect of UII in cardiovascular disease. Notably, to the best of our 
knowledge, the present results were the first to demonstrate that UII stimulation results in an inflammatory response, as shown by the increases in the levels of pro-inflammatory cytokines (TNF- $\alpha$ and IL-1 $\beta$ ) and the decreases in the levels of anti-inflammatory cytokines (IL-10 and MIF) in the serum of rats and the culture supernatant of VSMCs. Therefore, it was indicated that inflammatory factors induced by UII led to an inflammatory response that is involved in the pathological process of atherosclerosis.

RhoA and its downstream effector ROCK, which exists in two isoforms, ROCK1 and ROCK2, serve significant roles in multiple cellular processes, such as proliferation, apoptosis and migration (47). Abnormal activation of the RhoA/ROCK pathway has been reported to be involved in various types of diseases including diabetes, osteoarthritis, cardiovascular and cerebrovascular diseases (11-13). In recent years, the regulatory effect of the RhoA/ROCK pathway on the inflammatory process of atherosclerosis has been revealed. For instance, upregulation of the RhoA/ROCK signaling cascade has been identified in atherosclerosis (11). Moreover, RhoA-mediated $\mathrm{NF}-\kappa \mathrm{B}$ signaling pathways lead to vascular endothelial dysfunction in diabetes (48). ROCK pathways also contribute to hyperglycemia-activated macrophages, which result in a more pro-inflammatory phenotype and eventually lead to atherosclerosis (17). Previous studies have shown that ROCK I is predominantly increased in the process of macrophage adherence, and ROCK1-deficiency decreases atherosclerosis in bone marrow-derived cells (49), indicating that ROCK I serves an important role in the development of atherosclerosis. However, whether ROCK II inhibition could also be beneficial in attenuating atherosclerosis remain to be investigated. In line with these previous results, the present findings suggested that UII stimulation promoted the RhoA/ROCK pathway in rats and VSMCs, implying the involvement of the RhoA/ROCK II pathway in UII-induced inflammatory response.

It has been revealed that salidroside, an early antioxidant Tibetan medicine, has anti-inflammatory effects in atherosclerosis $(50,51)$. Li et al $(50)$ reported that salidroside can decrease the generation of inflammatory cytokines, such as IL-6, IL-1 $\beta$ and MCP-1, in TNF- $\alpha$-induced cardiac microvascular endothelial cells, alleviating vascular inflammation and atherosclerosis. Another previous study revealed that salidroside attenuated endothelial cellular senescence via reducing the expression of inflammatory cytokines, thus mitigating the pathogenesis of atherosclerosis (51). In addition, isorhamnetin, a flavonoid monomer extracted from seabuckthorn fruit, has been shown to possess anti-cancer, anti-oxidant, anti-inflammatory and anti-atherosclerotic activities $(34,52,53)$. However, there are few studies on the anti-inflammatory and anti-atherosclerotic effects of salidroside, isorhamnetin and both in combination.

To the best of our knowledge, the present study was the first to demonstrate that salidroside, isorhamnetin and both in combination decreased TNF- $\alpha$ and IL- $1 \beta$ levels, and increased IL-10 level in the serum of UII-treated rats and in the culture supernatant of UII-stimulated VSMCs, at concentrations corresponding to human therapeutic blood plasma concentrations, thus eliminating the UII-induced inflammatory response. High concentrations of salidroside and isorhamnetin both reversed the UII-induced inflammatory response in vivo and in vitro. However, the inhibitory effects of low concentration of salidroside and isorhamnetin on inflammatory response were inconsistent. The combination of low and high concentrations of salidroside and isorhamnetin both eliminated the UII-induced inflammatory response in vivo and in vitro. Furthermore, the anti-inflammatory effect of the combination of salidroside and isorhamnetin was not significantly different compared with the single drug alone during UII conditions in atherosclerosis.

Subsequently, based on the role of the RhoA/ROCK pathway in UII-induced inflammatory response, the present study further investigated the effects of salidroside, isorhamnetin and both in combination on the RhoA/ROCK pathway. It was found that salidroside, isorhamnetin and both in combination inhibited the RhoA/ROCK pathway in the thoracic aorta of rats following subacute infusion of UII and in UII-stimulated VSMCs. Consistent with the in vivo results, the inhibitory effects of low concentrations of salidroside and isorhamnetin on the RhoA/ROCK pathway were inconsistent, while the high concentrations of salidroside and isorhamnetin both reversed UII-induced the enhancement of the RhoA/ROCK pathway in vivo and in vitro. The combination of low and high concentrations of salidroside and isorhamnetin both attenuated the RhoA/ROCK pathway under UII in vivo and in vitro. There was no significant difference between salidroside and isorhamnetin in isolation and in combination. Thus, the results suggested that, to a certain extent, the RhoA/ROCK pathway contributed to the anti-inflammatory effects of salidroside, isorhamnetin and both in combination under UII simulation in atherosclerosis.

However, there are a few limitations to the present study. First, gain-of-function and loss-of function experiments were not used to investigate the role of the RhoA/ROCK pathway in UII-induced inflammatory or the anti-inflammatory effects of salidroside, isorhamnetin and both in combination. This will require further examination in future studies. In addition, the potential mechanism of salidroside, isorhamnetin and both in combination in the inhibition of the RhoA/ROCK pathway under UII has not yet been elucidated. The present study also did not examine the comparison of the two drugs with currently, widely-used anti-atherosclerosis drugs and other reported anti-inflammatory drugs in atherosclerosis, which may be interesting to evaluate in the subsequent experiment plan.

In conclusion, the present findings indicated that UII stimulation resulted in an inflammatory response, accompanied by the enhancement of the RhoA/ROCK pathway in vivo and in vitro. In addition, to the best of our knowledge, the present results provided the first evidence that salidroside, isorhamnetin and both in combination attenuated the UII-induced inflammatory response, which was partly dependent on inhibition of the RhoA/ROCK pathway. Moreover, there is no significant difference between salidroside and isorhamnetin, both in isolation or in combination. The present study may provide a novel theoretical basis for the separate used of the two drugs or their combination in the treatment against atherosclerosis. Furthermore, the present results may provide additional theoretical guidance for the clinical combined use of the Tibetan medicines salidroside and isorhamnetin in the prevention against atherosclerosis-related cardiovascular diseases. 


\section{Acknowledgements}

Not applicable.

\section{Funding}

The present study was supported by grants from National Natural Science Foundation of China (grant no. 81360490), research funds for Institutions of Higher Learning of Gansu Province (grant no. 2017B-81), the Fundamental Research Funds for the Central Universities (grant nos. 31920180023 and 31920190106) and the Natural Science Foundation of Gansu Province, China (grant no. 20JR5RA503).

\section{Availability of data and materials}

The datasets used and/or analyzed during the current study are available from the corresponding author on reasonable request.

\section{Authors' contributions}

$\mathrm{CW}$ and $\mathrm{XN}$ participated in the design of the study and conducted the experiment. CW, SP and YZ wrote the manuscript and analyzed data. XW, SM and GM collaborated in the analysis and interpretation of data. All authors read and approved the final manuscript.

\section{Ethics approval and consent to participate}

All animal experimental procedures were performed in accordance with the National Institutes of Health Guide for the Care and Use of Laboratory Animals, and were approved by the Experimental Animal Administration Committee of the School of Basic Medical Sciences, Northwest Minzu University Health Science Center (approval no. XBMZ-YX20130101; March 1, 2013).

\section{Patient consent for publication}

Not applicable.

\section{Competing interests}

The authors declare that they have no competing interests.

\section{References}

1. Libby P, Bornfeldt KE and Tall AR: Atherosclerosis: Successes, surprises, and future challenges. Circ Res 118: 531-534, 2016.

2. Chistiakov DA, Kashirskikh DA, Khotina VA, Grechko AV and Orekhov AN: Immune-inflammatory responses in atherosclerosis: The role of myeloid cells. J Clin Med 8: 1798, 2019.

3. Hanna A and Frangogiannis NG: Inflammatory cytokines and chemokines as therapeutic targets in heart failure. Cardiovasc Drugs Ther 34: 849-863, 2020.

4. Taleb S: Inflammation in atherosclerosis. Arch Cardiovasc Dis 109: 708-715, 2016.

5. Veluswamy P, Wacker M, Scherner M and Wippermann J: Delicate role of PD-L1/PD-1 axis in blood vessel inflammatory diseases: Current insight and future significance. Int J Mol Sci 21: 8159, 2020.

6. Sethwala AM, Goh I and Amerena JV: Combatting inflammation in cardiovascular disease. Heart Lung Circ 30: 197-206, 2021.
7. Koushki K, Shahbaz SK, Mashayekhi K, Sadeghi M, Zayeri ZD, Taba MY, Banach M, Al-Rasadi K, Johnston TP and Sahebkar A: Anti-inflammatory action of statins in cardiovascular disease: The role of inflammasome and toll-like receptor pathways. Clin Rev Allergy Immunol: May 6, 2020 (Epub ahead of print). doi: 10.1007/s12016-020-08791-9.

8. Luque-Martin R, Van den Bossche J, Furze RC, Neele AE, van der Velden S, Gijbels MJJ, van Roomen CPPA, Bernard SG, de Jonge WJ, Rioja I, et al: Targeting histone deacetylases in myeloid cells inhibits their maturation and inflammatory function with limited effects on atherosclerosis. Front Pharmacol 10: $1242,2019$.

9. Mog B, Asase C, Chaplin A, Gao H, Rajagopalan S and Maiseyeu A: Nano-antagonist alleviates inflammation and allows for MRI of atherosclerosis. Nanotheranostics 3: 342-355, 2019.

10. Yang G, Zhuo J, Lin Y, Zhang M, Liu L, Chen X and Gao R: Ginsenoside Rb1 prevents dysfunction of endothelial cells by suppressing inflammatory response and apoptosis in the high-fat diet plus balloon catheter-injured rabbit model via the $\mathrm{G}$ protein-coupled estrogen receptor-mediated phosphatidylinositol 3-kinases (PI3K)/akt pathway. Med Sci Monit 25: 7407-7417, 2019.

11. Cai A, Li L and Zhou Y: Pathophysiological effects of RhoA and Rho-associated kinase on cardiovascular system. J Hypertens 34: 3-10, 2016.

12. Deng Z, Jia Y, Liu H, He M, Yang Y, Xiao W and Li Y: RhoA/ROCK pathway: Implication in osteoarthritis and therapeutic targets. Am J Transl Res 11: 5324-5331, 2019.

13. Strzelecka-Kiliszek A, Mebarek S, Roszkowska M, Buchet R, Magne D and Pikula S: Functions of Rho family of small GTPases and Rho-associated coiled-coil kinases in bone cells during differentiation and mineralization. Biochim Biophys Acta Gen Subj 1861: 1009-1023, 2017.

14. Li N, Chen J, Zhao J and Wang T: MicroRNA-3188 targets ETS-domain protein 4 and participates in RhoA/ROCK pathway to regulate the development of atherosclerosis. Pharmazie 72: 687-693, 2017.

15. Surma M, Wei L and Shi J: Rho kinase as a therapeutic target in cardiovascular disease. Future Cardiol 7: 657-671, 2011.

16. Zhou Q, Gensch C and Liao JK: Rho-associated coiled-coil-forming kinases (ROCKs): Potential targets for the treatment of atherosclerosis and vascular disease. Trends Pharmacol Sci 32: 167-173, 2011.

17. Cheng CI, Chen PH, Lin YC and Kao YH: High glucose activates Raw264.7 macrophages through RhoA kinase-mediated signaling pathway. Cell Signal 27: 283-292, 2015.

18. Loirand G, Guerin P and Pacaud P: Rho kinases in cardiovascular physiology and pathophysiology. Circ Res 98: 322-334, 2006.

19. Shimada H and Rajagopalan LE: Rho-kinase mediates lysophosphatidic acid-induced IL-8 and MCP-1 production via p38 and JNK pathways in human endothelial cells. FEBS Lett 584: 2827-2832, 2010.

20. Desche P, Couderc LJ and Epardeau B: Sjogren's syndrome and pulmonary lymphangiomyomatosis. Chest $94: 898,1988$.

21. Douglas SA and Ohlstein EH: Human urotensin-II, the most potent mammalian vasoconstrictor identified to date, as a therapeutic target for the management of cardiovascular disease. Trends Cardiovasc Med 10: 229-237, 2000.

22. Albanese I, Daskalopoulou SS, Yu B, You Z, Genest J, Alsheikh-Ali A and Schwertani AG: The urotensin II system and carotid atherosclerosis: A role in vascular calcification. Front Pharmacol 7: 149, 2016.

23. Demirpence M, Guler A, Yilmaz H, Sayin A, Pekcevik Y, Turkon H, Colak A, Ari EM, Aslanipour B, Kocabas GU and Calan M: Is elevated urotensin II level a predictor for increased cardiovascular risk in subjects with acromegaly? J Endocrinol Invest 42: 207-215, 2019.

24. Şatıroğlu Ö, Durakoğlugil ME, Çetin M, Çiçek Y, Erdoğan T and Duman H: The role of urotensin II and atherosclerotic risk factors in patients with slow coronary flow. Interv Med Appl Sci 8: 158-163, 2016

25. Li Y, Zhao S, Wang Y, Chen Y, Lin Y, Zhu N, Zheng H, Wu M, Cheng D, Li Y, et al: Urotensin II promotes atherosclerosis in cholesterol-fed rabbits. PLoS One 9: e95089, 2014.

26. Lu D, Peng F, Li J, Zhao J, Ye X, Li B and Ding W: Urotensin II promotes secretion of LTB4 through 5-lipoxygenase via the UT-ROS-Akt pathway in RAW264.7 macrophages. Arch Med Sci 15: 1065-1072, 2019.

27. Xu S, Jiang $\mathrm{H}$, Wu B, Yang $\mathrm{J}$ and Chen S: Urotensin II induces migration of endothelial progenitor cells via activation of the RhoA/Rho kinase pathway. Tohoku J Exp Med 219: 283-288, 2009. 
28. Saito K, Yonekura-Sakakibara K, Nakabayashi R, Higashi Y, Yamazaki M, Tohge T and Fernie AR: The flavonoid biosynthetic pathway in arabidopsis: Structural and genetic diversity. Plant Physiol Biochem 72: 21-34, 2013.

29. Zhong Z, Han J, Zhang J, Xiao Q, Hu J and Chen L: Pharmacological activities, mechanisms of action, and safety of salidroside in the central nervous system. Drug Des Devel Ther 12: 1479-1489, 2018

30. Boesch-Saadatmandi C, Loboda A, Wagner AE, Stachurska A, Jozkowicz A, Dulak J, Döring F, Wolffram S and Rimbach G: Effect of quercetin and its metabolites isorhamnetin and quercetin-3-glucuronide on inflammatory gene expression: Role of miR-155. J Nutr Biochem 22: 293-299, 2011.

31. Lodi F, Jimenez R, Moreno L, Kroon PA, Needs PW, Hughes DA, Santos-Buelga C, Gonzalez-Paramas A, Cogolludo A, Lopez-Sepulveda R, et al: Glucuronidated and sulfated metabolites of the flavonoid quercetin prevent endothelial dysfunction but lack direct vasorelaxant effects in rat aorta. Atherosclerosis 204 34-39, 2009.

32. National Research Council (US): Committee for the Update of the Guide for the Care and Use of Laboratory Animals: Guide for the Care and Use of Laboratory Animals. 8th edition. National Academies Press, Washington, DC,2011.

33. Chen L, Liu P, Feng X and Ma C: Salidroside suppressing LPS-induced myocardial injury by inhibiting ROS-mediated PI3K/Akt/mTOR pathway in vitro and in vivo. J Cell Mol Med 21: 3178-3189, 2017.

34. Jamali-Raeufy N, Baluchnejadmojarad T, Roghani M, Keimasi S and Goudarzi M: Isorhamnetin exerts neuroprotective effects in STZ-induced diabetic rats via attenuation of oxidative stress, inflammation and apoptosis. J Chem Neuroanat 102: 101709, 2019.

35. Zhang N, Pei F, Wei H, Zhang T, Yang C, Ma G and Yang C: Isorhamnetin protects rat ventricular myocytes from ischemia and reperfusion injury. Exp Toxicol Pathol 63: 33-38, 2011.

36. Hwang SM, Lee YJ, Lee YP, Yoon JJ, Lee SM, Cha JD, Choi KM, Kang DG and Lee HS: Anti-proliferative effect of an aqueous extract of prunella vulgaris in vascular smooth muscle cells. Evid Based Complement Alternat Med 2013: 936463, 2013.

37. Livak KJ and Schmittgen TD: Analysis of relative gene expression data using real-time quantitative PCR and the 2(-Delta Delta C(T)) method. Methods 25: 402-408, 2001.

38. Zhou J, Yin G, Yu T, Zhang Y, Tian X, Xia D and Shi L: Rosuvastatin reduces expression of tissue factor through inhibiting RhoA/ROCK pathway to ameliorate atherosclerosis. Panminerva Med: Sep 24, 2019 (Epub ahead of print). doi: 10.23736/S00310808.19.03761-3.

39. Pereira-Castro J, Bras-Silva C and Fontes-Sousa AP: Novel insights into the role of urotensin II in cardiovascular disease. Drug Discov Today 24: 2170-2180, 2019.

40. Yu QQ, Cheng DX, Xu LR, Li YK, Zheng XY, Liu Y,Li YF,Liu HL, Bai L, Wang R, et al: Urotensin II and urantide exert opposite effects on the cellular components of atherosclerotic plaque in hypercholesterolemic rabbits. Acta Pharmacol Sin 41: 546-553, 2019.

41. Tsai CS, Loh SH, Liu JC, Lin JW, Chen YL, Chen CH and Cheng TH: Urotensin II-induced endothelin-1 expression and cell proliferation via epidermal growth factor receptor transactivation in rat aortic smooth muscle cells. Atherosclerosis 206 $86-94,2009$
42. Diebold I, Petry A, Burger M, Hess J and Görlach A: NOX4 mediates activation of FoxO3a and matrix metalloproteinase-2 expression by urotensin-II. Mol Biol Cell 22: 4424-4434, 2011.

43. Yang Y, Zhang J, Chen X, Wu T, Xu X, Cao G, Li H and Li Y: UII/GPR14 is involved in NF- $\kappa$ B-mediated colonic inflammation in vivo and in vitro. Oncol Rep 36: 2800-2806, 2016.

44. Fiordelisi A, Iaccarino G, Morisco C, Coscioni E and Sorriento D: NFkappaB is a key player in the crosstalk between inflammation and cardiovascular diseases. Int J Mol Sci 20: 1599, 2019.

45. Li Q, Zhao W, Zeng X and Hao Z: Ursolic acid attenuates atherosclerosis in apoE(-/-) mice: Role of LOX-1 mediated by ROS/NF- $\mathrm{B}$ pathway. Molecules 23: 1101-1108, 2018.

46. Zhang J, Wang X, Vikash V, Ye Q, Wu D, Liu Y and Dong W: ROS and ROS-mediated cellular signaling. Oxid Med Cell Longev 2016: 4350965, 2016.

47. Bustelo XR, Sauzeau V and Berenjeno IM: GTP-binding proteins of the Rho/Racfamily: Regulation, effectors and functions in vivo. Bioessays 29: 356-370, 2007.

48. Tang ST, Zhang Q, Tang HQ, Wang CJ, Su H, Zhou Q, Wei W, Zhu HQ and Wang Y: Effects of glucagon-like peptide-1 on advanced glycation endproduct-induced aortic endothelial dysfunction in streptozotocin-induced diabetic rats: Possible roles of Rho kinase- and AMP kinase-mediated nuclear factor $\kappa \mathrm{B}$ signaling pathways. Endocrine 53: 107-116, 2016.

49. Wang HW, Liu PY, Oyama N, Rikitake Y, Kitamoto S, Gitlin J, Liao JK and Boisvert WA: Deficiency of ROCK1 in bone marrow-derived cells protects against atherosclerosis in LDLR-/-mice. FASEB J 22: 3561-3570, 2008.

50. Li R, Dong Z, Zhuang X, Liu R, Yan F, Chen Y, Gao X and Shi H: Salidroside prevents tumor necrosis factor- $\alpha$-induced vascular inflammation by blocking mitogen-activated protein kinase and NF- $\kappa B$ signaling activation. Exp Ther Med 18: 4137-4143, 2019.

51. Xing SS, Li J, Chen L, Yang YF, He PL, Li J and Yang J: Salidroside attenuates endothelial cellular senescence via decreasing the expression of inflammatory cytokines and increasing the expression of SIRT3. Mech Ageing Dev 175: 1-6, 2018.

52. Luo Y, Sun G, Dong X, Wang M, Qin M, Yu Y and Sun X: Isorhamnetin attenuates atherosclerosis by inhibiting macrophage apoptosis via PI3K/AKT activation and HO-1 induction. PLoS One 10: e120259, 2015.

53. Park C, Cha HJ, Choi EO, Lee H, Hwang-Bo H, Ji SY, Kim MY, Kim SY, Hong SH, Cheong JH, et al: Isorhamnetin induces cell cycle arrest and apoptosis via reactive oxygen species-mediated AMP-activated protein kinase signaling pathway activation in human bladder cancer cells. Cancers (Basel) 11: 1494, 2019.

This work is licensed under a Creative Commons Attribution-NonCommercial-NoDerivatives 4.0 International (CC BY-NC-ND 4.0) License. 